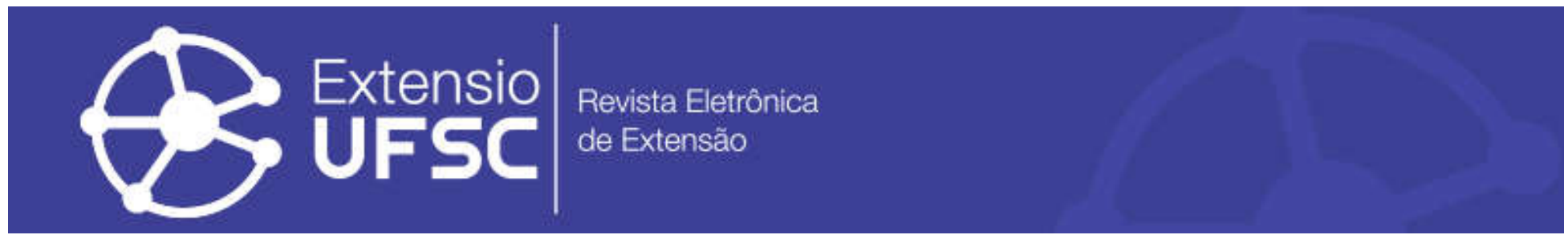

\title{
AÇÃO EDUCACIONAL PROMOVIDA POR ESTUDANTES DE FARMÁCIA EM UM CENTRO ONCOLÓGICO DE MOSSORÓ/RN
}

\author{
José Nyedson Moura de Góis \\ Faculdade Nova Esperança de Mossoró \\ josenyedson@hotmail.com \\ Luanne Eugênia Nunes \\ Faculdade Nova Esperança de Mossoró \\ luanne_87@hotmail.com
}

Resumo

Este trabalho objetiva relatar a construção e o desenvolvimento, bem como mensurar os resultados de uma ação social em saúde promovida por discentes do curso de Farmácia em um centro oncológico filantrópico da cidade de Mossoró/RN em setembro de 2019, que abordou o uso racional de medicamentos, enfatizando fitoterápicos e plantas medicinais. Para a intervenção, além da comunicação verbal, dispôs-se de materiais gráficos, como folders e slides, além de formulações farmacêuticas fitoterápicas, como um gel à base de Aloe vera e sachês contendo flores de camomila. A ação proporcionou aos alunos conhecer in loco uma área específica de atuação farmacêutica no âmbito do cuidado ao paciente e a integração de conceitos e metodologias ativas.

Palavras-chave: Promoção da Saúde. Assistência Farmacêutica. Oncologia. Fitoterapia. Plantas Medicinais.

\section{EDUCATION ACTION PROMOTED BY PHARMACY STUDENTS IN THE ONCOLOGY CENTER OF MOSSORÓ/RN}

\begin{abstract}
This work aims report the construction and the development, as well measure the results of a health social activity promoted by Pharmacy's students at a philanthropic oncological center in the Mossoró/RN city in september 2019, that discourse about rational medicine use, lay emphasis on phytotherapics and medicinal plants. For the intervention, besides the verbal communication, was used graphic materials, such as folders and slides, besides pharmaceutical herbal formulations such as an Aloe vera-based gel and sachets containing chamomile flowers. The activity provided students to learn in loco a specific area of pharmaceutical activity within the scope of patient care and the integration of concepts and active methodologies.

Keywords: Health Promotion. Pharmaceutical Services. Medical Oncology. Phytotherapy. Medicinal Plants.

\section{ACCIÓN EDUCATIVA PROMOVIDO POR ESTUDIANTES DE LA CARRERA DE FARMACIA EN UN CENTRO ONCOLÓCO DEL MOSSORÓ/RN}

\section{Resumen}

Este trabajo tiene como objetivo informar la construcción y el desarrollo, bien como medir los resultados de una acción social en salud promovida por estudiantes de la carrera de Farmacia en um centro oncológico filantrópico em la ciudad de Mossoró/RN en septiembre de 2019, en donde se pude abordar el uso racional de medicamentos, haciendo hincapié en los fitoterápicos y las plantas medicinales. Para la intervención, además de la comunicación verbal, estaban disponibles materiales gráficos, como cartalesas y diapositivas, además de formulaciones farmacéuticas a base de hierbas, como um gel que tiene como a base de Aloe vera y sobres que conteniendo flores de manzanilla. La acción pudo brindar a los estudiantes la oportunidad de aprender in loco acerca de un área específica de actividad farmacéutica dentro del alcance de la atención al paciente y la integración de conceptos y metodologias activas.

Palavras clave: Promoción de la Salud. Servicios Farmacéuticos. Oncología Médica. Fitoterapia. Plantas Medicinales. 
Ação educacional promovida por estudantes de Farmácia em um centro oncológico de Mossoró/RN

\section{INTRODUÇÃO}

Considera-se câncer o crescimento ininterrupto e desordenado de células em uma determinada região, com potencial de agredir também tecidos e órgãos adjacentes ou não, existindo mais de 100 tipos da condição (INCA, 2019).

Trata-se de uma doença multifatorial, podendo ser intrínsecos (genética) ou extrínsecos (radiação, substâncias e parasitos) ao organismo (OPAS, 2018). Estima-se que cerca de 80 a 90\% dos casos de câncer no Brasil envolvam causas externas. Além disso, estatísticas do Instituto Nacional de Câncer (2019) apontam uma expectativa de aproximadamente 625 mil casos entre homens e mulheres para 2020, sendo o último grupo de maior incidência, o que evoca certo receito pela sociedade (INCA, 2019).

Nessa perspectiva, é cada vez mais intensa a busca por medidas cautelares, na tentativa de minimizar principalmente os efeitos dos fatores externos (OPAS, 2018). Assim, o farmacêutico, um dos profissionais da saúde que perpassa por vários níveis assistenciais, deve promover seu envolvimento e inserção efetiva na prática direta do cuidado com o paciente, e não apenas na manipulação e dispensação de medicamentos, passando, deste modo, a colaborar com a melhoria da qualidade de vida do indivíduo (CALADO et al., 2019).

A Resolução do Conselho Federal de Farmácia (CFF) nº 288, de 1996, determinou ser privativa ao farmacêutico a manipulação de substâncias citotóxicas. Posteriormente, o Ministério da Saúde, em 1998, ainda atribuiu a atuação do farmacêutico como indispensável para serviços de alta complexidade, como a oncologia. Contudo, este profissional continua se adaptando ao referido cenário, trazendo suas contribuições na área clínico-assistencial (ANDRADE, 2009).

As atribuições clínicas do farmacêutico estão descritas na Resolução do CFF no 585 de 2013, permitindo-o atuar na busca ativa dos problemas relacionados aos medicamentos (PRM), para evitar, reduzir ou eliminar resultados negativos a medicamentos (RNM), através do acompanhamento farmacoterapêutico direto ou indireto, minimizando erros de prescrição e aparições de reações adversas a medicamentos (RAM), apresentando consequente melhora clínica e aceitação do paciente, posto a eficácia e segurança do tratamento oncológico (CFF, 2013; CALADO et al., 2019).

No que tange ao tratamento oncológico, este é considerado um método inespecífico, haja vista que as substâncias não atuam apenas no local desejado, desenvolvendo, consequentemente, reações adversas que interferem na qualidade de vida do paciente (INCA, 2019).

Com o surgimento de RAM, muitas vezes graves, é necessário dispor de recursos terapêuticos adjuvantes. As terapias complementares visam combinar-se aos tratamentos 
Ação educacional promovida por estudantes de Farmácia em um centro oncológico de Mossoró/RN

convencionais, e não os substituir (FUKUMASU et al., 2008; INCA, 2019). Nesse sentido, Rodrigues e Gusman (2018) observaram quais são as práticas secundárias mais utilizadas, apontando a fitoterapia como a principal delas.

Apesar de ser uma terapia milenar, o uso irracional da fitoterapia e de plantas medicinais também podem gerar danos ao organismo, assim como os medicamentos alopáticos. Portanto, pacientes oncológicos configuram-se como grupo de risco para o desenvolvimento de reações adversas devido à polifarmácia, isto é, uso simultâneo de dois ou mais medicamentos (FUKUMASU et al., 2008).

Visto isso, o ensino universitário, em especial na área da saúde, deve integrar-se a esses ambientes comunitários na tentativa de somar com seus processos de pesquisa ao conhecimento social, desempenhando seu papel na construção da sociedade. Ademais, tais formatos metodológicos permitem aos alunos participar como sujeito ativo, contextualizando e interpretando suas experiências práticas de aproximação com cenários de atuação, os quais são mecanismos indispensáveis para promover uma reflexão educacional, profissional e humanitária (BRANDÃO; ROCHA; SILVA, 2013; RABELO et al., 2016).

Nos municípios do Rio Grande do Norte (RN), o uso de plantas medicinais para o tratamento e prevenção de agravos de saúde é muito prevalente (COELHO et al., 2016; CORDEIRO; BOTREL; HOLANDA, 2017). Desse modo, a equipe de saúde da instituição visitada, a partir de evidencias clínicas, prescreve determinadas plantas medicinais como adjuvantes para o tratamento tópico de implicações da radioterapia (GOMES, 2010).

No entanto, os pacientes oncológicos constantemente fazem uso de outras espécies vegetais, na forma de formulações fitoterápicas caseiras e sem embasamento técnico-científico, visando amenizar os efeitos colaterais do tratamento oncológico, ou até mesmo o próprio agravo da condição (CAETANO et al., 2015). Assim, é quase que imensurável a dimensão do uso irracional de plantas medicinais por esse grupo de pacientes, os quais já são submetidos a tratamentos alta complexidade.

À vista disso, propôs-se uma ação em saúde diante da necessidade da comunidade em ter um momento educacional acerca do uso de medicamentos, uma vez que a prática da automedicação é constante, de modo a auxiliar o entendimento dessa tecnologia em saúde tão utilizada, favorecendo o controle e a prevenção do uso irracional e de possíveis efeitos secundários.

Dessa forma, com este trabalho, objetivou-se relatar a construção de uma ação comunitária em saúde centrada no cuidado farmacêutico ao paciente com câncer, bem como mensurar os resultados de uma ação social em saúde promovida por discentes do curso de 
Ação educacional promovida por estudantes de Farmácia em um centro oncológico de Mossoró/RN

Farmácia em um centro oncológico filantrópico da cidade de Mossoró/RN, o Hospital da Solidariedade de Mossoró (HSM), em setembro de 2019.

\section{MATERIAIS E MÉTODOS}

Esta ação em saúde surgiu como parte da disciplina Seminários Integradores e Ensino/Pesquisa/Comunidade V (SIESC V), sendo a ocorrência primitiva da quinta edição pela instituição de ensino promotora. Este componente visa integrar conhecimentos teóricos e práticos adquiridos ao longo das outras edições e dos componentes curriculares obrigatórios do período e aplicá-los (BRANDÃO; ROCHA; SILVA, 2013; FACENE, 2019). Neste caso, enfatizou-se o uso da fitoterapia e de plantas medicinais, intervindo por meio de práticas assistenciais farmacêuticas, conhecida como Atenção Farmacêutica ou Cuidados Farmacêuticos, envolvendo ainda conceitos e técnicas da farmácia de manipulação e de farmacognosia.

Este estudo possui caráter descritivo e qualitativo, sendo decorrente de uma experiência de extensão universitária vivenciada pelos discentes do quinto período do curso de graduação em Farmácia da Faculdade Nova Esperança de Mossoró (Facene/RN).

A ação foi desenvolvida no Hospital da Solidariedade de Mossoró (HSM), sendo esta uma unidade especializada em oncologia e referência local no tratamento por radioterapia. O HSM presta serviços à comunidade desde 2013 e é vinculado à Liga Mossoroense de Estudo e Combate ao Câncer (LMECC), uma instituição não governamental e sem fins lucrativos que oferta assistência multidisciplinar e integrada aos pacientes oncológicos (LMECC, 2018).

Em relação à demanda do hospital, no que tange ao serviço de atendimento clínicoassistencial, tem-se uma rotatividade de pacientes ligeiramente alta, uma vez que o HSM atende não somente pacientes da cidade a qual está situado, mas abarca inclusive pacientes das demais regiões interioranas do Estado, além de outras cidades do Nordeste. Apenas em 2016, a LMECC atendeu 1.143 novos pacientes. No entanto, esses dados são generalizados, isto é, abarca os dois hospitais especializados em oncologia vinculados à LMECC, não havendo menção apenas para os pacientes do Hospital da Solidariedade em específico (LMECC, 2018).

Quanto à instituição de saúde, o HSM dispõe de uma farmácia de manipulação, nomeada de Gotas de Solidariedade, que oferta medicamentos de alto custo a cerca de 1.000 pacientes cadastrados. Internamente, é dividida segundo as formas farmacêuticas produzidas, tais como: sólidos, líquidos e semissólidos. Há duas farmacêuticas atuantes na instituição, ambas prestam assistência direta aos pacientes, além das atribuições nos setores de produção e de controle de 
Ação educacional promovida por estudantes de Farmácia em um centro oncológico de Mossoró/RN

qualidade, principalmente. Contudo, há uma baixa procura dos serviços voltados ao cuidado farmacêutico por parte dos pacientes.

No que diz respeito aos serviços da farmácia de manipulação Gotas da Solidariedade, Kaliane Soares, Gestora do HSM destaca: "Atendemos por volta de 90 pacientes diariamente. Eles realizam um cadastro, e, de acordo com a oferta de medicamentos, recebem na farmácia, tanto os pacientes da radioterapia, quanto os de outras unidades da LMECC, basta ser paciente e estar devidamente cadastrado" (PRECISAMOS REFERENCIAR).

Durante a ação no HSM, foram abordados temas como: conceitos fundamentais sobre fármacos e medicamentos; diferenciação entre medicamentos genéricos, equivalentes e de referência; armazenamento de medicamentos; descarte de medicamentos; automedicação; uso racional de medicamentos alopáticos, fitoterápicos e plantas medicinais; métodos básicos de obtenção de derivados vegetais (processos extrativos por infusão e decocção); interações medicamentosas; interferências em exames clínicos; reações adversas à medicamentos (RAM); importância da prática de exercícios físicos.

Não houve processo de seleção e divisão por faixa etária, sexo ou qualquer outro critério de classificação, pois a ação foi desenvolvida em pleno horário de funcionamento da citada instituição, a qual recepciona tanto pacientes quanto seus familiares e/ou cuidadores que os acompanham nos procedimentos.

Para o planejamento, etapa que antecede a ação propriamente dita, foram realizadas reuniões diárias para delinear os conteúdos a serem trabalhados, atividades, metodologias e objetivos da ação. Além disso, houve uma visita prévia às edificações do hospital pela professora Dra. Luanne Eugênia Nunes, para acordar com os gestores da instituição a possibilidade do desenvolvimento da ação e quais as necessidades gerais dos pacientes quanto às informações em saúde e a eventual data para execução da intervenção.

Como solicitado, houve permissão da instituição para que os discentes pudessem efetivar o SIESC V, que ocorreu entre os dias 23 e 27 de setembro de 2019. Os 3 primeiros dias foram destinados ao planejamento da ação por parte dos alunos, uma vez que os funcionários do Hospital, o que incluem as farmacêuticas, não participaram do processo de confecção dos materiais, nem do planejamento e organização da ação, deixando-a inteiramente sobre responsabilidade dos alunos e da professora supracitada, a qual participou como supervisora. Além disso, houve 1 dia para a ação in loco e 1 dia para o feedback com a turma, conforme detalhado no Quadro 1.

Inicialmente, houve uma contextualização sobre a oncologia e os centros oncológicos, em especial o HSM e a LMECC, com a docente responsável por subsidiar os alunos na ação. Para 
Ação educacional promovida por estudantes de Farmácia em um centro oncológico de Mossoró/RN

isso, se fez uso de metodologias expositivas, dando sugestões sobre a forma na qual os assuntos deveriam ser abordados e trabalhados, além de apresentar as formulações fitoterápicas e magistrais comumente prescritas aos pacientes.

\begin{tabular}{|c|c|c|}
\hline DATA & TURNO & TAREFA \\
\hline $\begin{array}{l}\text { Segunda-feira, } \\
23 / 09 / 2019\end{array}$ & Noite & $\begin{array}{l}\text { - Explanação teórica acerca da oncologia (o que é, causas, } \\
\text { tratamento e efeitos adversos), da LMECC e do HSM; } \\
\text { - Objetivos da ação; } \\
\text { - Idealizou-se a confecção de um folder informativo; } \\
\text { - Determinou-se um coffee break; } \\
\text { - Cogitou-se a manipulação de um gel de Aloe vera. }\end{array}$ \\
\hline \multirow{3}{*}{$\begin{array}{l}\text { Terça-feira, } \\
\text { 24/09/2019 }\end{array}$} & Manhã & - Iniciou-se a produção do folder. \\
\hline & Tarde & $\begin{array}{l}\text { - Orçamento dos materiais (folder, alimentos, extrato de Aloe } \\
\text { vera e embalagens). }\end{array}$ \\
\hline & Noite & $\begin{array}{l}\text { - Cotação coletiva; } \\
\text { - Cogitou-se produzir sachês de camomila para distribuição; } \\
\text { - Deu-se continuidade a produção do folder. }\end{array}$ \\
\hline \multirow{3}{*}{$\begin{array}{l}\text { Quarta-feira, } \\
25 / 09 / 2019\end{array}$} & Manhã & $\begin{array}{l}\text { - Adquiriu-se as embalagens, o extrato glicólico de Aloe vera, } \\
\text { a flor da camomila e os alimentos. }\end{array}$ \\
\hline & Tarde & $\begin{array}{l}\text { - Impressão dos folders; } \\
\text { - Realizou-se a assepsia das embalagens; } \\
\text { - Manipulação do gel de Aloe vera. }\end{array}$ \\
\hline & Noite & $\begin{array}{l}\text { - } \quad \text { Envase do gel; } \\
\text { - } \quad \text { Produção dos sachês de camomila; } \\
\text { - Impressão e aplicação dos respectivos rótulos; } \\
\text { - } \quad \text { Dobraram-se os folders; } \\
\text { - Criou-se uma apresentação em slides. }\end{array}$ \\
\hline $\begin{array}{l}\text { Quinta-feira, } \\
\text { 26/09/2019 }\end{array}$ & Manhã & - Dia da ação. \\
\hline $\begin{array}{l}\text { Sexta-feira, } \\
27 / 09 / 2019\end{array}$ & Noite & - Reunião coletiva. \\
\hline
\end{tabular}

Fonte: elaborado pelos autores, 2019.

Embasando-se nisso, foi idealizado a produção de materiais gráficos utilizando recursos digitais para que os ouvintes pudessem entender o que de fato seria levantado na ação. Foram elaboradas apresentações em slides, por meio do Microsoft PowerPoint ${ }^{\circledR}$, versão 2019, com figuras e imagens contendo principalmente informações acerca do uso racional de medicamentos. Além disso, se confeccionou folders informativos, utilizando o Microsoft Publisher ${ }^{\circledR}$, também na versão 2019, contendo informações primordiais sobre as plantas medicinais utilizadas. 
Ação educacional promovida por estudantes de Farmácia em um centro oncológico de Mossoró/RN

Vale ressaltar que todos os materiais desenvolvidos foram adaptados à linguagem dita popular, evitando termos e conceitos específicos, deixando as informações descritas mais claras e objetivas.

Por fim, como forma de agradecimento, quanto a futuros profissionais farmacêuticos, decidiu-se manipular um gel à base de Aloe vera (babosa) para os pacientes, semelhante ao formulado pela farmácia Gotas da Solidariedade. Ainda houve uma oferta de sachês de Matricaria chamomilla (camomila), fracionados e pesados pelos alunos. Ambas as plantas são indicadas como terapia complementar, aos pacientes, pelos profissionais da referida instituição.

\section{RESULTADOS E ANÁLISES}

Como mencionado, à medida que era idealizado um método de intervenção educativa, este seria elaborado. Com isso, resultou-se na concretização de todas as tarefas propostas: elaboração de recursos didáticos e metodológicos (folders e slides), manipulação do gel, confecção dos sachês e realização do coffee break para os ouvintes.

No tocante aos produtos obtidos, em suma, as formulações preparadas foram bem aceitas pelo grupo de pacientes e pelos profissionais do hospital, incluindo as farmacêuticas, que abordaram os alunos com dúvidas sobre o preparo e a eficácia destas apresentações.

A obtenção do gel à base do extrato glicólico de Aloe vera (Figura 1a, 1b e 1c) se deu no laboratório de farmacotécnica da faculdade de origem, sob supervisão da mencionada Professora Dra. Luanne Eugênia Nunes, a qual partilha a autoria deste trabalho, e com materiais adquiridos de forma particular em laboratórios farmacêuticos de referência da região. Baseando-se na Farmacopeia Brasileira, 6a ed. (BRASIL, 2019), respeitou-se a seguinte formulação: extrato glicólico de Aloe vera (10\%), base de carbopol (q.s.p.), conservante, corante e essência (ambos em q.s.).

Figura 1 -(a) Gel de Aloe vera manipulado, (b) embalagens pós-assepsia, (c) produto acondicionado e rotulado e sachê de (d) Matricaria chamomilla rotulado.

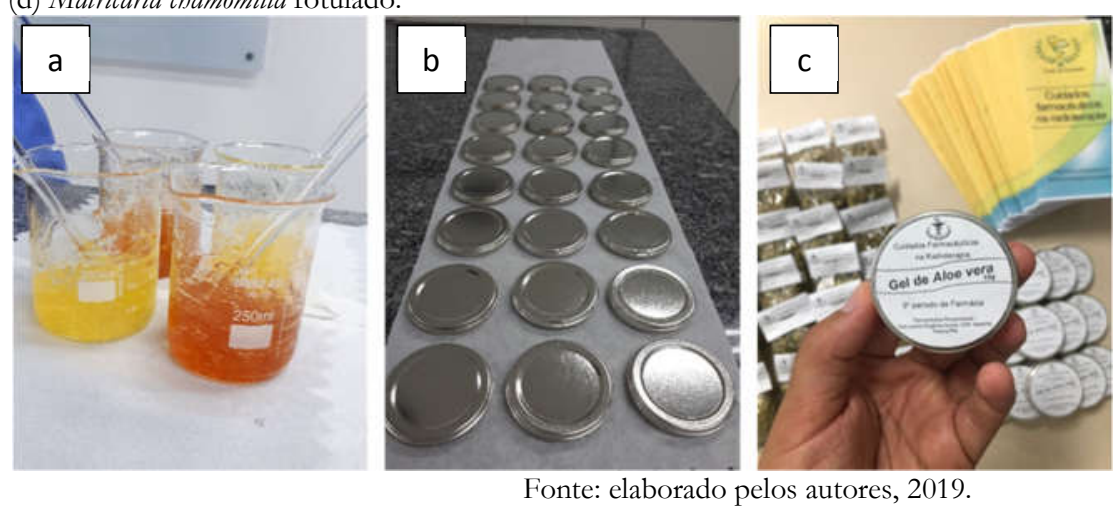

Fonte: elaborado pelos autores, 2019.

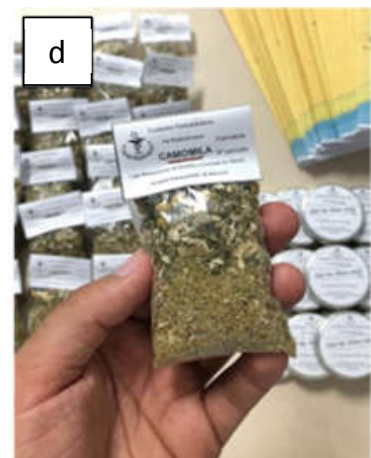


Ação educacional promovida por estudantes de Farmácia em um centro oncológico de Mossoró/RN

Ainda se confeccionou sachês contendo aproximadamente $15 \mathrm{~g}$ de droga vegetal Matricaria chamomilla (Figura 1d) para que os pacientes preparassem, em suas respectivas residências, e sob as orientações contidas no folder e abordadas na palestra, um chá por infusão para compressas nos locais de lesão, conforme indicação dos prescritores.

Estas formulações são habitualmente prescritas pelos profissionais do citado centro oncológico, sendo indicadas para tratamento e prevenção de lesões oriundas da radioterapia. Logo, não houve nenhum tipo de prescrição ou indicação por partes dos estudantes, havendo exclusivamente orientação quanto ao preparo e uso racional.

Estudos demonstram que espécies como Aloe vera e Matricaria chamomilla são eficazes para o tratamento de lesões cutâneas (ARSIĆ et al., 2011; BEIKERT et al., 2013). As propriedades farmacológicas da Matricaria chamomillapara o manejo de diferentes lesões na pele é vinculado a substâncias como terpenos e flavonoides oriundos do metabolismo secundários. A redução no aparecimento de eritemas e a inibição do processo inflamatório, por via das cicloxigenases, são propriedades mencionadas por Ferreira et al. (2015) e, dentre as indicações, encontra-se o tratamento e prevenções de radiodermites (SCHNEIDER et al., 2013).

De forma semelhante, também houve descrição da atividade da Aloe vera, principalmente no tratamento dessas implicações da radioterapia, devido aos seus efeitos anti-inflamatórios e cicatrizantes vinculados aos polissacarídeos acemanana e manose-6-fosfato encontrados no gel (FREITAS; RODRIGUES; GASPI, 2014). Tais informações justificam o emprego das formulações fitoterápicas, que são frequentemente propostas se indicadas em vários centros de oncologia (SCHNEIDER et al., 2013).

O público ouvinte foi bastante variado, constituindo-se principalmente de adultos e idosos. Alguns profissionais e estagiários da instituição também se juntaram ao momento. O quantitativo total foi superior a 80 participantes, em fluxo contínuo, onde muitos retornaram após realizar as sessões de radioterapia e/ou consultas. Uma grande parcela participou ativamente da palestra e demonstrou interesse nos assuntos abordados, realizando perguntas pertinentes de modo a dinamizar ainda mais a forma como foi ministrada a temática.

À vista disso, o processo de aprendizagem por parte dos discentes foi constante, pois, a cada questionamento, surgia uma nova forma de intervenção a qual não havia sido planejada. Portanto, foram resgatados conteúdos teórico-práticos vistos em outros componentes, além daqueles trabalhados durante a palestra. Dessa forma, os alunos demonstraram rapidez, concisão e acessibilidade no decorrer da intervenção. 
Ação educacional promovida por estudantes de Farmácia em um centro oncológico de Mossoró/RN

Assim discorre Rabelo et al. (2016), enfatizando que a extensão universitária possibilita justamente essa interação, envolvendo o estudante, o professor, o conteúdo, a prática, o contexto e a comunidade, permitindo-o desenvolver competências sociais, postura profissional e senso crítico durante essas ações, contribuindo ainda como progresso da qualidade educacional devido à inserção em ambientes sociais externos.

Os recursos digitais utilizados para projetar figuras, imagens e informações textuais favoreceram a compreensão do assunto, pois, durante a palestra, alguns dos pacientes mencionaram não possuir desenvoltura adequada apenas para leitura e interpretação. Desse modo, o folder (Figura 2) foi bem aceito por servir de recurso dinâmico e de fácil acesso a ser utilizado posteriormente por seus cuidadores e/ou familiares. Ainda, uma cerca quantia do folder confeccionado pelos estudantes ficou disponível na recepção do hospital, para que os pacientes e/ou seus acompanhantes que não participaram da ação pudessem ter acesso ao conteúdo tratado no decorrer das suas sucessivas idas ao local.

Durante toda a palestra, os pacientes abordaram os discentes com dúvidas relacionadas principalmente com: armazenamento e descarte adequado de medicamentos; automedicação; reações adversas à medicamentos; interações medicamentosas; fracionamento de medicamentos; manejo de posologia; e formas de preparo de chás. Ainda foram levantadas temáticas não programadas como: uso e interações dos contraceptivos orais; utilização de antibióticos e resistência bacteriana; e mecanismo de ação e reações adversas da quimioterapia.

Figura 2 - Representação das partes externa e interna dofolder, respectivamente.
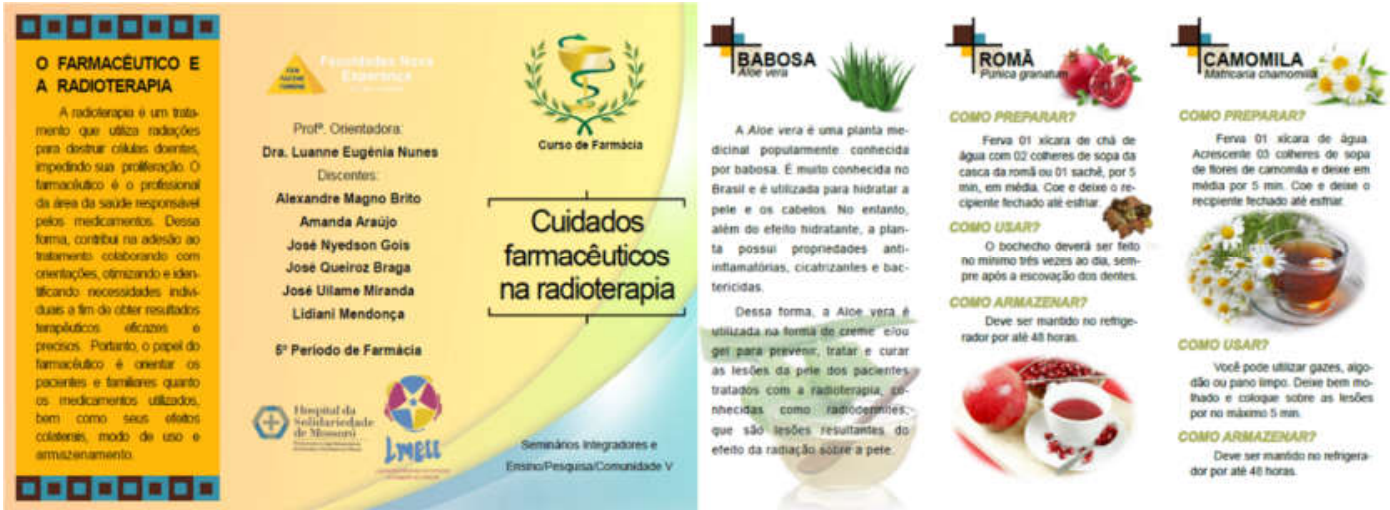

Fonte: elaborado pelos autores, 2019.

Além disso, de forma espontânea, os pacientes apontaram questionamentos relacionado ao respectivo tratamento, bem como suas possíveis reações adversas, assuntos que deveriam ser tratados e discutidos durante consultas com os profissionais que os assistem. Porém, durante suas 
Ação educacional promovida por estudantes de Farmácia em um centro oncológico de Mossoró/RN

falas, os pacientes narraram sentir-se receosos em fazer esses questionamentos devido à demanda elevada de consultas, que as tornam curtas e objetivas.

Assim, notou-se que os pacientes incluíam em suas narrativas a internet como fonte de informação mais recorrida para sanar eventuais dúvidas. Sabe-se que esse mecanismo de busca, quando bem empregada, pode ser útil. No entanto, por se tratar de leigos no que diz respeito à oncologia, pode ser falho, identificando métodos de tratamentos que por muitas vezes não são comunicados aos profissionais, podendo diminuir a eficácia da terapia oncológica e/ou culminar no surgimento de outras reações.

Por meio dessas pesquisas, descreveram encontrar métodos de tratamento e de prevenção do câncer a partir de plantas medicinais e/ou medicamentos fitoterápicos. Em síntese, o uso dessas apresentações foi justificado como “o que é natural não faz mal”, associado às inúmeras indicações descritas nas plataformas, ou ainda mencionado por outras pessoas.

Em relação a isso, Fukumasu et al. (2008) avaliaram as principais espécies vegetais que, quando administradas concomitantemente, causam interação medicamentosa com os fármacos antineoplásicos convencionais. Dentre as espécies citadas que constam evidências contra as associações estão: Echinacea purpúrea, Maytenus ilicifolia, Panax ginseng e Hiperricum perforatum. Contudo, concluíram não haver estudos suficientes sobre as demais espécies com o grau de complexidade exigido pelo assunto, inclusive algumas são utilizadas pelos pacientes.

Apesar disso, sabe-se que os componentes ativos, ou seja, com propriedades medicinais são resultantes do metabolismo secundário desses vegetais. No entanto, estes dependem de fatores relacionados ao cultivo, solo, tempo de coleta, espécie, ambiente, dentre outros, além do método extrativo apropriado para a substância de interesse. Não havendo esse controle, as plantas podem desenvolver outros produtos talvez não desejados que exerçam efeitos mais tóxicos, por exemplo, justificando exatamente o não uso (GOBBO-NETO; LOPES, 2007).

De tal maneira, atividades educacionais externas como esta devem objetivar não apenas informação e divulgação do conhecimento, mas também implicar no desenvolvimento de posturas que colaborem com a mudança e adaptação das ações do ouvinte, desconstruindo pressupostos e concepções errôneas através do estímulo ao diálogo, à reflexão e ao questionamento. Além disso, a transformação pessoal do sujeito só é possível quando há envolvimento, empenho e aceitação em relação à temática, o que é favorecido pelo trabalho e discussão coletiva (VINHOLES; ALANO; GALATO, 2009).

No encerramento da ação, os pacientes se sentiram confortáveis para compartilhar com grupo algumas das reações adversas que mais os atingiam. Relataram em consonância àquelas mencionadas por Pinho et al. (2016) e ao que está descrito nas diretrizes do INCA (2019). As 
Ação educacional promovida por estudantes de Farmácia em um centro oncológico de Mossoró/RN

RAM mais comuns foram: náusea, vômito, constipação, diarreia, cefaleia, eritema, mucosite, radiodermite, hiperpigmentação e alopecia. Também descreveram quadros anêmicos, de toxicidade renal, cardíaca, pulmonar e neural.

Diante disso, nota-se que os pacientes oncológicos fazem uso de inúmeros medicamentos, classificando-os como indivíduos susceptíveis a desenvolverem RAM, principalmente pelos aspectos da polifarmácia, além dos tratamentos diretos relacionados ao câncer. Todavia, embora cientes de tal consequência, uma grande parcela dos pacientes apontou adicionar à sua terapêutica plantas medicinais de forma independente, baseando-se nas indicações da internet, de familiares e/ou de colegas. E também demonstraram omitir a administração conjunta destes com a terapia convencional, resultando em um aspecto que deve ser debatido frequentemente.

Neste sentido, intervenções baseadas na educação em saúde se fazem muito relevantes, uma vez que incluem a aplicação de políticas públicas a partir de propostas pedagógicas com o objetivo de promover a qualidade de vida e estimular o pensamento crítico dos pacientes. A educação em saúde é uma ferramenta que pode auxiliar a minimizar ou impedir a ocorrência de eventos relacionados a medicamentos decorrentes, por exemplo, do uso indevido das plantas medicinais (MENDIETA et al., 2014).

Ademais, o farmacêutico é o profissional de saúde que, na maioria das vezes, tem contato com os pacientes fora do ambiente do serviço básico de saúde. Por esta razão, deve promover ações na comunidade que incentivem os indivíduos a buscarem informações seguras, colaborando com a educação em saúde e uso racional de medicamentos, principalmente, o que acaba tornando essas atividades educacional de caráter público ferramentas e estratégias essenciais na promoção da saúde.

Por fim, alguns dos profissionais do hospital mencionaram a grandiosidade e significância em receber ações desta dimensão em unidades hospitalares, principalmente filantrópicas, as quais são alicerçadas em ações e doações da comunidade e demais órgãos de domínio público ou privado. Também citaram que apesar da vontade de promover momentos de educação em saúde de forma coletiva, nem sempre é possível devido à demanda do próprio hospital. Assim, evidenciou-se que há possibilidades de novas ações, em específico para as demais turmas do curso de Farmácia da instituição, que certamente passarão pelo SIESC V. 
Ação educacional promovida por estudantes de Farmácia em um centro oncológico de Mossoró/RN

\section{CONSIDERAÇÕES FINAIS}

A atenção humanizada é importante em todas as áreas do cuidado à saúde e é de fundamental importância em doenças graves, como o câncer. Na oncologia, a integração de uma equipe multiprofissional a partir de amplos e efetivos canais de comunicação torna a terapêutica mais eficaz, segura e bem tolerada pelos pacientes.

Nessa perspectiva, este projeto obteve êxito e mérito, tanto dos pacientes, que relataram certa carência de informações relacionados aos assuntos tratados, quanto institucional, pois como é visto em vários eixos e níveis de saúde, a relação entre demanda de atendimento elevada e reduzido quantitativo de profissionais de saúde dificulta a prática de educação em saúde nessa dimensão.

Notou-se uma abertura maior dos pacientes para se expressar e dialogar com os estudantes. Fato este pode ser explicado pelo ambiente social no qual o paciente está inserido, pois o formato de palestra pode ter agregado ao momento uma sensação de leveza e de fluidez como em uma "roda de conversa".

A importância dada às plantas medicinais e fitoterápicos é praticamente indiscutível, visto que diante da singular fragilidade psicológica e emocional que se encontra cada paciente, a incansável busca por tratamentos que possam gerar segurança, conforto e, principalmente, esperança, torna-se um objetivo coletivo, unificado.

Portanto, a desmistificação de que muitas espécies são a verdadeira "cura" é tarefa fundamental e de difícil execução, pois o paradigma de que "Plantas são naturais. Nada natural faz mal" está absurdamente impregnado na sociedade.

Ainda é necessário apresentá-los à dimensão desses atos e que essa busca pode ser mediada por profissionais aptos. Cabe aos pesquisadores avaliar a possibilidade dessas interações medicamentosas, já que a maioria dos pacientes utilizam preparações à base de plantas.

Por fim, o comprometimento dos alunos, o reconhecimento dos profissionais e a satisfação e o entusiasmo dos pacientes estimularam os gestores educacionais a reconhecer a importância e a necessidade de tais atividades, que geram interesse, aprendizado, reconhecimento e estímulo de ambas as partes, colaborando ainda com o sistema de saúde pública.

\section{AGRADECIMENTOS}

Agradecemos aos futuros farmacêuticos da turma de Farmácia 2021.1: Alexandre Brito, Amanda Araújo, José Queiroz Braga, José Uilame Cardozo e Lidiani Mendonça, pelo suporte 
Ação educacional promovida por estudantes de Farmácia em um centro oncológico de Mossoró/RN

durante a elaboração, o planejamento e a execução da atividade prevista. À Facene/RN por disponibilizar prontamente um de seus laboratórios de farmácia para manipulação da formulação, além de dar-nos a oportunidade de desempenhar tal extensão. Ao coordenador de curso, Me. Emanuell Santos, por idealizar parte do projeto conosco. Aos gestores e profissionais do Hospital da Solidariedade de Mossoró/RN pela receptividade e aceite do desenvolvimento desta ação. Aos pacientes e acompanhantes pela atenção, colaboração e participação. Mantenham-se firmes e conscientes.

\section{REFERÊNCIAS}

ARSIĆ, I.et al. Preparation of novel apigenin-enriched, liposomal and non-liposomal, antiinflammatory topical formulations as substitutes for corticosteroid therapy. PhytotherapyResearch, v. 25, n. 2, p. 228-233, 2011.

BAZANTE, P. H. S. Terapia da dor para pacientes oncológicos. Monografia (Especialização em Farmácia Hospitalar e Clínica) - Centro de Capacitação Educacional (CCE). Recife, 2016.

BEIKERT, F. C. et al. Antiinflammatory potential of seven plant extracts in the ultraviolet erythema test. A randomized, placebo-controlled study. Der Hautarzt; Zeitschriftfur Dermatologie, Venerologie, undverwandte Gebiete, v. 64, n. 1, p. 40, 2013.

BRANDÃO, E. R. M.; ROCHA, S. V.; SILVA, S. S. Práticas de integração Ensino-ServiõComunidade: reorientando a formação médica. Revista Brasileira de Educação Médica. v. 37, n. 4, p.573-577, set. 2013.

BRASIL. Agência Nacional de Vigilância Sanitária. Farmacopeia Brasileira, volume 1. $6^{\mathrm{a}}$ ed. Brasília: Anvisa, 2019. 874p.

CAETANO, N. L. B.et al. Plantas medicinais utilizadas pela população do município de Lagarto-SE, Brasil-ênfase em pacientes oncológicos. Revista Brasileira de Plantas Medicinais, v. 17, n. 4, p. 748-756, 2015.

CALADO, D. S.; TAVARES, D. H. C.; BEZERRA, G. C.O papel da atenção farmacêutica na redução das reações adversas associadas ao tratamento de pacientes oncológicos. Revista Brasileira de Educação e Saúde. Pombal/PB, v. 9, n. 3, p.94-99, set. 2019.

COELHO, M. D. F. B. et al. Levantamento etnobotânico das espécies vegetais em quintais de bairro na cidade de Mossoró, Rio Grande do Norte. Revista Verde de Agroecologia e Desenvolvimento Sustentável, v. 11, n. 4, p. 154-162, 2016.

CORDEIRO, M. C.; BOTREL, R. T.; HOLANDA, A. C. Levantamento etnobotânico de espécies arbóreas no assentamento Tabuleiro Grande, Apodi, Rio Grande do Norte. Revista Verde de Agroecologia e Desenvolvimento Sustentável, v. 12, n. 1, p. 122-131, 2017.

FACENE. Faculdade Nova Esperança de Mossoró. Projeto Pedagógico do Curso de Graduação em Farmácia. Mossoró/RN, 2019. 
Ação educacional promovida por estudantes de Farmácia em um centro oncológico de Mossoró/RN

FERREIRA, E. B.et al. Topical effects of Chamomilla recutita in skin damage: a literature review. Pharmacology Online, v. 3, p.123-130, dez. 2015.

FREITAS, V. S.; RODRIGUES, R. A. F.; GASPI, F. O. G. Pharmacological activities of Aloe vera (L.) Burm. f. Revista brasileira de plantas medicinais, v. 16, n. 2, p. 299-307, 2014.

FUKUMASU H.et al. Fitoterápicos e potenciais interações na terapia medicamentosa do câncer. Revista Brasileira de Toxicologia, v. 21, n. 2, p.49-59, 2008.

GOBBO-NETO, L.; LOPES, N. P. Plantas medicinais: fatores de influência no conteúdo de metabólitos secundários. Quím. Nova, São Paulo, v. 30, n. 2, p. 374-381, abr. 2007.

GOMES, P. B. B.S.. Uso da Aloe vera na profilaxia de radiodermite em pacientes portadoras de câncer de mama submetidas à cirurgia e tratadas com radioterapia adjuvante. Dissertação (Mestrado em Oncologia) - Programa de Pós-Graduação Interinstitucional (MINTER) em Oncologia da Fundação Antônio Prudente (FAP) em parceria com a Escola Cearense de Oncologia (ECO). Fortaleza, 2010.

INCA. Instituto Nacional de Câncer José Alencar Gomes da Silva. Estimativa 2020: incidência de câncer no Brasil / Instituto Nacional de Câncer José Alencar Gomes da Silva. - Rio de Janeiro: INCA, 2019. 120 p.: il. color (versão eletrônica).

KUMAR, V.; ABBAS, A.; ASTER, J.; Robbins \& Cotran. Patologia: bases patológicas das doenças. Com ilustrações de James A. Perkinns. 9. ed. Rio de Janeiro: Elsevier Editora Ltda., 2016.

MENDIETA, M. D. C. et al. Toxic plants: importance of knowledge for realization of health education. Journal of Nursing UFPE/Revista de Enfermagem UFPE, v. 8, n. 3, 2014.

OPAS. Organização Pan-Americana da Saúde. Folha informativa - Câncer. Brasil: OPAS, 2018. Disponível em: https://www.paho.org/bra/ Acesso em fev. 2020.

PASTORE, M. N. Processos de formação e cenários de ensino-aprendizagem: discussão sobre práticas em saúde e educação em serviço no curso de graduação em terapia ocupacional da FMUSP. Editora Cubo[s.1.], v. 26, n. 2, p. 431-441, 2018.

PINHO, M. S.; ABREU, Paula Alvarez; NOGUEIRA, Thaisa Amorim. Atenção farmacêutica a pacientes oncológicos: uma revisão integrativa da literatura. Revista Brasileira de Farmácia Hospitalar e Serviços de Saúde, São Paulo, v. 7, n. 1, p.33-39, mar. 2016.

RABELO, M. O. et al. O mutirão de prevenção ao câncer: um relato de experiência no âmbito da extensão universitária. Revista Intercâmbio, v. 7, p. 406-413, 2016.

RODRIGUES, C. L.; GUSMAN, G. S. Uso de terapias alternativas e complementares por pacientes oncológicos em quimioterapia, Revista Científica Univiçosa, v. 10, n. 1, p.315-320, dez. 2018.

SCHINEIDER, F.et al. Prevenção e tratamento de radiodermatite: uma revisão integrativa. Cogitare Enferm[s.l.], v. 18, n. 3, p.579-586, jul. 2013. 
Ação educacional promovida por estudantes de Farmácia em um centro oncológico de Mossoró/RN

SILVA, L. C. A. et al. Contribuições da atenção farmacêutica á pacientes em tratamento oncológico. Revista de Investigação Biomédica. São Luiz, v. 9, n. 2, p.210-217, mai. 2017.

VINHOLES, E. R.; ALANO, G. M.; GALATO, D.A percepção da comunidade sobre a atuação do serviço de atenção farmacêutica em ações de educação em saúde relacionadas à promoção do uso racional de medicamentos. Saúde Soc. São Paulo, v.18, n.2, p.293-303, 2009.

Recebido em: 02/07/2020

Aceito em: 01/04/2021 